\title{
Editorial
}

\section{Peer Review Week 2018: Diversity in Peer Review}

I look forward to Peer Review Week because, first, it gives me a chance to thank publicly the volunteer reviewers who are critical members of The Journal of Neuroscience community. Each year, you agree to read and evaluate the work submitted to JNeurosci, and your participation in this process makes the work even stronger. Thank you for the work you do. As we move to many parallel and diverse ways to get scientific discovery out into the community, I want to affirm my commitment, and the commitment of JNeurosci, to the peer review process and to the idea that we are willing to come together to agree on what controls and standards provide the basis on which neuroscience knowledge can move forward. Like all human endeavors, peer review is not perfect, but the consensus opinion of reviewers knowledgeable in the field, along with oversight by committed editors, provides an incredibly important service to the scientific enterprise. As in past years, we will be honoring a few of our most insightful and relied-upon reviewers with certificates of appreciation and a public shout-out, if they don't mind being acknowledged.

Second, each year there is a different theme for Peer Review Week that highlights a key issue in the process. This year the theme is "Diversity in Peer Review." At JNeurosci, this means many different things. First, it means making sure that the breadth of the authors who submit their work to JNeurosci is reflected among the reviewers who evaluate their work. Diversity at every level is important to make sure that the consensus on what makes a strong study reflects a broad definition of excellence. More than half of the papers submitted to JNeurosci are from outside the United States, and we work to make sure that the pool of reviewers is equally representative of our global authorship. We have only recently begun keeping records of gender for both our authors and reviewers, so our analyses are still incomplete and preliminary, but among those papers for which we have data, a little more than one-third of our sub- missions have a female corresponding author, and those papers have an equal (actually, slightly better) rate of acceptance compared with papers with a male corresponding author. The percentage of female reviewers lags behind corresponding authors slightly, however, and we are focused on making sure that this ratio catches up by sharing names of female reviewers and prompting reviewers who decline to think of female (and non-U.S.) scientists when they make suggestions for alternative reviewers to invite.

Finally, we are committed to making sure that the next generation of peer reviewers has the tools they need to provide constructive, fair, and rigorous reviews, and that they have a pathway into the review process at JNeurosci. One way we are working to do this is to call on our Associate Editors, who are chosen for their commitment to the peer review process shown by their outstanding, rapid, and frequent reviews for JNeurosci, to provide training to trainees through our Reviewer Mentoring Program (http:// www.jneurosci.org/content/38/3/511). This is a pilot; and because it involves one-on-one interactions, it can only accommodate a subset of trainees who would like to be involved, but we are interested in ideas on how we can scale up this program and include more trainees and mentors. Both trainees and mentors have enjoyed the program, and you can read some of their comments here: http:// neuronline.sfn.org/Articles/Professional-Development/2018/ The-Importance-of-Diversity-and-Inclusion-in-Peer-Review.

As always, if you have suggestions on how we can improve the peer review process at JNeurosci, please e-mail me at JN_EIC@SfN.org or tweet @MarinaP63.

(D) Marina Picciotto

EiC JNeurosci

DOI:10.1523/JNEUROSCI.2072-18.2018 